\title{
Global stability of Cohen-Grossberg neural network with both time-varying and continuous distributed delays
}

\author{
José J. Oliveira \\ Departamento de Matemática e Aplicações and CMAT, Escola de Ciências, \\ Universidade do Minho, Campus de Gualtar, 4710-057 Braga, Portugal \\ e-mail: jjoliveira@math.uminho.pt
}

\begin{abstract}
In this paper, a generalized neural network of Cohen-Grossberg type with both discrete time-varying and distributed unbounded delays is considered. Based on M-matrix theory, sufficient conditions are established to ensure the existence and global attractivity of an equilibrium point. The global exponential stability of the equilibrium is also addressed but for the model with bounded discrete time-varying delays. A comparison of results shows that these results generalize and improve some earlier publications.
\end{abstract}

Keywords: Cohen-Grossberg neural network, unbounded delay, time-varying delay, distributed delay, global asymptotic stability, global exponential stability.

2010 Mathematics Subject Classification: 34K20, 92B20.

\section{Introduction}

The Cohen-Grossberg neural network models, first proposed and studied by Cohen and Grossberg [4], have been the subject of an active research due to their large application in various engineering and scientific areas such as neural-biology, population biology, and computing technology. The neural network in [4] can be described by the following system of ordinary differential equations

$$
\dot{x}_{i}(t)=-a_{i}\left(x_{i}(t)\right)\left[b_{i}\left(x_{i}(t)\right)-\sum_{j=1}^{n} c_{i j} f_{j}\left(x_{j}(t)\right)+I_{i}\right], \quad i=1, \ldots, n .
$$

In order to be more realistic, differential equations describing neural networks should incorporate time delays to take into account physical and biological phenomena. It is known that time delays may lead to oscillation, divergence, or instability to a system [14].

For over two decades, several generalizations of model (1.1) with constant, discrete timevarying, or continuous distributed delays have been proposed and studied (see [2], [3], [18], [19], [20], and references therein). Besides the cited works, there is an extensive literature dealing with stability of Cohen-Grossberg models with delays.

Recently, neural network models with both discrete time-varying and continuous distributed delays have been considered [17], [21], and the following Cohen-Grossberg neural network model

$$
\begin{aligned}
\dot{x}_{i}(t)=-a_{i}\left(x_{i}(t)\right)\left[b_{i}\left(x_{i}(t)\right)-\sum_{j=1}^{n}\right. & c_{i j} g_{j}\left(x_{j}(t)\right)-\sum_{j=1}^{n} d_{i j} f_{j}\left(x_{j}\left(t-\tau_{i j}(t)\right)\right) \\
& \left.-\sum_{j=1}^{n} q_{i j} \int_{-\infty}^{0} k_{i j}(-s) v_{j}\left(x_{j}(t+s)\right) d s+I_{i}\right], \quad t \geq 0
\end{aligned}
$$


was studied in [13], [16].

In this paper we consider a generalization of (1.2), see (2.1) below, and shall study the existence of an equilibrium point and its global attractivity and global exponential stability. We emphasize that, contrary to the usual approach in the literature, instead of Lyapunov functional techniques, we apply some quite different techniques, presented in [5], [6], [15]) to study the global stability of an equilibrium point of the model. Our method allows us to deal with more general models.

Now we set some definitions and notations. We denote by $B C=B C\left((-\infty, 0] ; \mathbb{R}^{n}\right)$ the space of bounded and continuous functions, $\phi:(-\infty, 0] \rightarrow \mathbb{R}^{n}$, equipped with the norm $\|\phi\|_{\infty}=\sup _{s \leq 0}|\phi(s)|$, where $|\cdot|$ is the maximum norm in $\mathbb{R}^{n}$, i.e. $|x|=\max \left\{\left|x_{i}\right|: i=1, \ldots, n\right\}$ for $x=\left(x_{1}, \ldots, x_{n}\right) \in \mathbb{R}^{n}$. For $a \in \mathbb{R}^{n}$, we also use $a$ to denote the constant function $\varphi(s)=a$ in $B C$. A vector $c=\left(c_{1}, \ldots, c_{n}\right) \in \mathbb{R}^{n}$ is said to be positive if $c_{i}>0$ for $i=1, \ldots, n$ and in this case we write $c>0$. For a real matrix $A=\left[a_{i j}\right]_{n \times n}$ we denote by $|A|$ the absolute-value matrix given by $|A|=\left[\left|a_{i j}\right|\right]_{n \times n}$.

For an open set $D \subseteq B C$ and $f:[0,+\infty) \times D \rightarrow \mathbb{R}^{n}$ continuous, consider the functional differential equation (FDE) given in a general setting by

$$
\dot{x}(t)=f\left(t, x_{t}\right), \quad t \geq 0,
$$

where, as usual, $x_{t}$ denotes the function $x_{t}:(-\infty, 0] \rightarrow \mathbb{R}^{n}$ defined by $x_{t}(s)=x(t+s)$ for $s \leq 0$. As we are interested in neural network models, we always consider solutions of (1.3) with initial bounded conditions.

It is well-known that the Banach space $B C$ is not an admissible phase space for (1.3) in the sense of [10], consequently the standard existence, uniqueness, continuous dependence type results are not available. Instead of $B C$, we consider the admissible Banach space

$$
U C_{g}=\left\{\phi \in C\left((-\infty, 0] ; \mathbb{R}^{n}\right): \sup _{s \leq 0} \frac{|\phi(s)|}{g(s)}<\infty, \frac{\phi(s)}{g(s)} \text { is uniformly continuous on }(-\infty, 0]\right\},
$$

equipped with the norm $\|\phi\|_{g}=\sup _{s \leq 0} \frac{|\phi(s)|}{g(s)}$, where $g:(-\infty, 0] \rightarrow[1, \infty)$ is a function satisfying:

(g1) $g$ is a non-increasing continuous function and $g(0)=1$;

(g2) $\lim _{u \rightarrow 0^{-}} \frac{g(s+u)}{g(s)}=1$ uniformly on $(-\infty, 0]$;

(g3) $g(s) \rightarrow+\infty$ as $s \rightarrow-\infty$.

As $B C \subseteq U C_{g}$, then $B C$ is a subspace of $U C_{g}$, and we denote by $B C_{g}$ the space $B C$ with the norm $\|\cdot\|_{g}$.

As $U C_{g}$ is an admissible Banach space, we consider the FDE (1.3), in the phase space $U C_{g}$, for a convenient function $g$ and under enough smooth properties of $f$, and we have existence and uniqueness of solution for the initial value problem (see [11]). We denote by $x(t, 0, \varphi)$ the solution of (1.3) with initial condition $x_{0}=\varphi, \varphi \in U C_{g}$.

In section 2, we establish sufficient conditions for the existence and global attractivity of an equilibrium point of a general Cohen-Grossberg neural network model. An equilibrium point $x^{*}$ of a FDE is said to be globally attractive if any solution $x(t)$ with bounded initial condition satisfies $x(t) \rightarrow x^{*}$ as $t \rightarrow+\infty$. 
In section 3, we set sufficient conditions for the equilibrium point to be globally exponentially stable. An equilibrium point $x^{*}$ of a neural network model is said to be globally exponentially stable if there are positive constants $\varepsilon, M$ such that

$$
\left|x(t, 0, \varphi)-x^{*}\right| \leq M e^{-\varepsilon t}\left\|\varphi-x^{*}\right\|_{\infty} \quad \forall t \geq 0, \varphi \in B C .
$$

As mentioned in [7], the definition of global exponential stability of an equilibrium $x^{*}$ is the usual in the literature on neural networks with unbounded delays, but is does not even imply the stability of $x^{*}$ in the phase space $U C_{g}$, i.e. relative to the norm $\|\cdot\|_{g}$.

To prove the boundedness solutions of the differential systems studied in this paper, we use the following result established in [7].

Lemma 1.1. [7] Consider equation (1.3) in $U C_{g}$, and suppose that $f$ transforms closed bounded sets of $[0,+\infty) \times D$ into bounded sets of $\mathbb{R}^{n}$. If the hypothesis

(H) for all $t \geq 0$ and $\varphi \in B C$ such that $|\varphi(s)|<|\varphi(0)|$ for $s \in(-\infty, 0)$, then $\varphi_{i}(0) f_{i}(t, \varphi)<$ 0 for some $i \in\{1, \ldots, n\}$ such that $|\varphi(0)|=\left|\varphi_{i}(0)\right|$

holds, then any solution $x(t)$ of (1.3) with initial condition $\varphi \in B C$ is defined and bounded on $[0,+\infty)$, and verifies $|x(t)| \leq\|\varphi\|_{\infty}$ for all $t \geq 0$.

To prove the existence and uniqueness of an equilibrium point, we make use of arguments in [15] and the following lemma:

Lemma 1.2. [9] If $H: \mathbb{R}^{n} \rightarrow \mathbb{R}^{n}$ is a continuous and injective function such that

$$
\lim _{|x| \rightarrow+\infty}|H(x)|=+\infty,
$$

then $H(x)$ is a homeomorphism of $\mathbb{R}^{n}$ onto itself.

\section{Global attractivity}

Consider the generalized Cohen-Grossberg model with both discrete time-varying and continuous distributed infinite delays given by

$$
\begin{aligned}
\dot{x}_{i}(t)=-a_{i}\left(x_{i}(t)\right)\left[b_{i}\left(x_{i}(t)\right)+\sum_{j=1}^{n} \sum_{p=1}^{P}\right. & \left(h_{i j}^{(p)}\left(x_{j}\left(t-\tau_{i j}^{(p)}(t)\right)\right)+\right. \\
& \left.\left.+f_{i j}^{(p)}\left(\int_{-\infty}^{0} g_{i j}^{(p)}\left(x_{j}(t+s)\right) d \eta_{i j}^{(p)}(s)\right)\right)\right], t \geq 0
\end{aligned}
$$

$i=1, \ldots, n$, where $a_{i}: \mathbb{R} \rightarrow(0, \infty), b_{i}, h_{i j}^{(p)}, f_{i j}^{(p)}, g_{i j}^{(p)}: \mathbb{R} \rightarrow \mathbb{R}, \tau_{i j}^{(p)}:[0, \infty) \rightarrow[0, \infty)$ are continuous functions, and $\eta_{i j}^{(p)}:(-\infty, 0] \rightarrow \mathbb{R}$ are non-decreasing bounded functions, normalized so that $\eta_{i j}^{(p)}(0)-\eta_{i j}^{(p)}(-\infty)=1$, for all $i, j \in\{1, \ldots, n\}, p \in\{1, \ldots, P\}$. In [7], a function $g:(-\infty, 0] \rightarrow[1,+\infty)$ was defined by

(i) $g(s)=1$ on $\left[-r_{1}, 0\right]$;

(ii) $g\left(-r_{n}\right)=n, n \in \mathbb{N}$;

(iii) $g$ is continuous and piecewise linear (linear on intervals $\left[-r_{n+1},-r_{n}\right]$ ), 
where $r_{n} \nearrow+\infty$ is a suitable sequence of positive numbers, in such a way that conditions (g1), (g2), (g3) hold and

$$
\int_{-\infty}^{0} g(s) d \eta_{i j}^{(p)}<+\infty, \quad i, j=1, \ldots, n, p=1, \ldots, P .
$$

See more details in Lemma 4.1 in [7]. Thus, we may consider the differential system (2.1) in the phase space $U C_{g}$.

As mentioned above, we only consider solutions of (2.1) with bounded initial data, i.e.,

$$
x_{0}=\varphi, \varphi \in B C .
$$

In the sequel, for (2.1) the following hypotheses will be considered:

(A1) for each $i \in\{1, \ldots, n\}$, there is $\beta_{i}>0$ such that

$$
\left(b_{i}(u)-b_{i}(v)\right) /(u-v) \geq \beta_{i}, \quad \forall u, v \in \mathbb{R}, u \neq v ;
$$

(A2) $h_{i j}^{(p)}, f_{i j}^{(p)}, g_{i j}^{(p)}: \mathbb{R} \rightarrow \mathbb{R}$ are Lipschitz functions with Lipschitz constants $\gamma_{i j}^{(p)}, \mu_{i j}^{(p)}$, and $\sigma_{i j}^{(p)}$, respectively, for $i, j=1, \ldots, n, p=1, \ldots, P$

(A3) $t-\tau_{i j}^{(p)}(t) \rightarrow \infty$ as $t \rightarrow \infty$, for $i, j=1, \ldots, n, p=1, \ldots, P$.

Now, we define the square real matrices,

$$
B=\operatorname{diag}\left(\beta_{1}, \ldots, \beta_{n}\right), \quad L=\left[l_{i j}\right] \quad \text { and } \quad N=B-L,
$$

where $\beta_{1}, \ldots, \beta_{n}$ are as in (A1) and $l_{i j}=\sum_{p=1}^{P} \gamma_{i j}^{(p)}+\mu_{i j}^{(p)} \sigma_{i j}^{(p)}$.

We recall here the definition of a non-singular M-matrix. For further properties of Mmatrices, we refer the reader to Chapter 5 of [8].

Definition. If $D=\left[d_{i j}\right]$ is a square matrix with non-positive off-diagonal entries, i.e., $d_{i j} \leq 0$ for all $i \neq j$, we say that $D$ is a non-singular $M$-matrix if all the eigenvalues of $D$ have positive real part, or, equivalently, if all the principal minors of $D$ are positive.

Theorem 2.1. Assume (A1)-(A3). For $N$ defined in (2.3), if $N$ is a non-singular $M$ matrix, then there is a unique equilibrium point $x^{*}=\left(x_{1}^{*}, \ldots, x_{n}^{*}\right)$ of (2.1) which is globally attractive.

Proof. Since $N$ is a non-singular M-matrix, then (see [8]) there is $d=\left(d_{1}, \ldots, d_{n}\right)>0$ such that $N d>0$, i.e.,

$$
\beta_{i}>d_{i}^{-1}\left(\sum_{j=1}^{n} l_{i j} d_{j}\right), \quad i=1, \ldots, n .
$$

The change $z_{i}(t)=d_{i}^{-1} x_{i}(t)$ transforms $(2.1)$ into

$$
\dot{z}_{i}(t)=-\bar{a}_{i}\left(z_{i}(t)\right)\left[\bar{b}_{i}\left(z_{i}(t)\right)+h_{i}\left(t, z_{t}\right)\right], \quad t \geq 0, \quad i=1, \ldots, n,
$$


where, for each $t \geq 0, i=1, \ldots, n, \phi \in U C_{g}$, and $u \in \mathbb{R}$, we have

$$
\begin{aligned}
& h_{i}(t, \phi)=d_{i}^{-1}\left[\sum_{j=1}^{n} \sum_{p=1}^{P}\left(h_{i j}^{(p)}\left(d_{j} \phi_{j}\left(-\tau_{i j}^{(p)}(t)\right)\right)+f_{i j}^{(p)}\left(\int_{-\infty}^{0} g_{i j}^{(p)}\left(d_{j} \phi_{j}(s)\right) d \eta_{i j}^{(p)}(s)\right)\right)\right], \\
& \bar{a}_{i}(u)=a_{i}\left(d_{i} u\right), \quad \bar{b}_{i}(u)=d_{i}^{-1} b_{i}\left(d_{i} u\right) .
\end{aligned}
$$

Note that $\left(\bar{b}_{i}(u)-\bar{b}_{i}(v)\right) /(u-v) \geq \beta_{i}$ for $u, v \in \mathbb{R}, u \neq v$, i.e., condition (A1) is satisfied by the functions $\bar{b}_{i}, i \in\{1, \ldots, n\}$.

Define the continuous function $H: \mathbb{R}^{n} \rightarrow \mathbb{R}^{n}$,

$$
H(x)=\left(\bar{b}_{i}\left(x_{i}\right)+d_{i}^{-1} \sum_{j=1}^{n} \sum_{p=1}^{P}\left(h_{i j}^{(p)}\left(d_{j} x_{j}\right)+f_{i j}^{(p)}\left(g_{i j}^{(p)}\left(d_{j} x_{j}\right)\right)\right)\right)_{i=1}^{n}, x=\left(x_{1}, \cdots, x_{n}\right) \in \mathbb{R}^{n} .
$$

Then $x^{*} \in \mathbb{R}^{n}$ is an equilibrium of the neural network (2.5) if and only if $H\left(x^{*}\right)=0$. Consequently, to prove the existence and uniqueness of an equilibrium point, it is sufficient to prove that $H$ is a homeomorphism. Arguing as in the proof of Lemma 2.4 in [15], we conclude that $H$ is injective and $\lim _{|x| \rightarrow \infty}|H(x)|=\infty$, thus, from Lemma 1.2, we obtain that $H$ is a homeomorphism, hence there is a unique equilibrium point $x^{*} \in \mathbb{R}^{n}$ of (2.5).

Translating the equilibrium to the origin through the change $y_{i}(t)=z_{i}(t)-x_{i}^{*},(2.5)$ becomes

$$
\dot{y}(t)=f\left(t, y_{t}\right), \quad t \geq 0,
$$

where $f=\left(f_{1}, \ldots, f_{n}\right):[0, \infty) \times U C_{g} \rightarrow \mathbb{R}^{n}$ is defined by

$$
f_{i}(t, \phi)=-\bar{a}_{i}\left(\phi_{i}(0)+x_{i}^{*}\right)\left[\bar{b}_{i}\left(\phi_{i}(0)+x_{i}^{*}\right)+h_{i}\left(t, \phi+x^{*}\right)\right] .
$$

It is easy to see that $f$ transforms closed bounded sets of $[0,+\infty) \times U C_{g}$ into bounded sets of $\mathbb{R}^{n}$.

Let $t \geq 0$ and $\varphi \in B C$ satisfying $|\varphi(s)|<|\varphi(0)|$, for $s \in(-\infty, 0)$, and let $i \in\{1, \ldots, n\}$ be such that $|\varphi(0)|=\left|\varphi_{i}(0)\right|$. Suppose that $\varphi_{i}(0)>0$ (the situation is analogous for $\varphi_{i}(0)<0$ ). Then $\varphi_{i}(0)=\sup _{s \leq 0}|\varphi(s)|$ and

$$
\begin{aligned}
& \bar{b}_{i}\left(\varphi_{i}(0)+x_{i}^{*}\right)+h_{i}\left(t, \varphi+x^{*}\right)=\bar{b}_{i}\left(\varphi_{i}(0)+x_{i}^{*}\right)-\bar{b}_{i}\left(x_{i}^{*}\right) \\
& +d_{i}^{-1} \sum_{j=1}^{n} \sum_{p=1}^{P}\left[\left(h_{i j}^{(p)}\left(d_{j} \varphi_{j}\left(-\tau_{i j}^{(p)}(t)\right)+d_{j} x_{j}^{*}\right)-h_{i j}^{(p)}\left(d_{j} x_{j}^{*}\right)\right)\right. \\
& \left.\quad+f_{i j}^{(p)}\left(\int_{-\infty}^{0} g_{i j}^{(p)}\left(d_{j} \varphi_{j}(s)+d_{j} x_{j}^{*}\right) d \eta_{i j}^{(p)}(s)\right)-f_{i j}^{(p)}\left(g_{i j}^{(p)}\left(d_{j} x_{j}^{*}\right)\right)\right] \\
& \geq \beta_{i} \varphi_{i}(0)-d_{i}^{-1} \sum_{j=1}^{n} \sum_{p=1}^{P}\left(\gamma_{i j}^{(p)} d_{j}\left|\varphi_{j}\left(-\tau_{i j}^{(p)}(t)\right)\right|+\mu_{i j}^{(p)} \sigma_{i j}^{(p)} d_{j} \int_{-\infty}^{0}\left|\varphi_{j}(s)\right| d \eta_{i j}^{(p)}(s)\right) \\
& \geq \beta_{i} \varphi_{i}(0)-d_{i}^{-1} \sum_{j=1}^{n} l_{i j} d_{j} \sup _{s \leq 0}|\varphi(s)|=\left(\beta_{i}-d_{i}^{-1} \sum_{j=1}^{n} l_{i j} d_{j}\right) \sup _{s \leq 0}|\varphi(s)|>0,
\end{aligned}
$$


hence $f$ satisfies hypothesis $\mathbf{( H )}$. Consequently, for $y(t)=\left(y_{i}(t)\right)_{i=1}^{n}$ a solution of $(2.6)$ with initial condition $y_{0} \in B C$, from Lemma 1.1 we conclude that $y(t)$ is defined and bounded on $\mathbb{R}$ and verifies

$$
|y(t)| \leq\left\|y_{0}\right\|_{\infty}, \quad t \geq 0
$$

Set

$$
-v_{i}=\liminf _{t \rightarrow \infty} y_{i}(t), \quad u_{i}=\limsup _{t \rightarrow \infty} y_{i}(t), \quad i=1, \ldots, n
$$

and

$$
v=\max _{i}\left\{v_{i}\right\}, \quad u=\max _{i}\left\{u_{i}\right\} .
$$

Note that $u, v \in \mathbb{R}$ and $-v \leq u$.

It is sufficient to prove that $\max \{u, v\}=0$. Assume e.g. that $|v| \leq u$, so that $\max \{u, v\}=$ $u$. (The situation $|u| \leq v$ is analogous). Let $i \in\{1, \ldots, n\}$ such that $u_{i}=u$.

Arguing as in the proof of Theorem 3.2 in [7], we can conclude that there is a positive real sequence $\left(t_{k}\right)_{k \in \mathbb{N}}$ such that

$$
t_{k} \nearrow \infty, \quad y_{i}\left(t_{k}\right) \rightarrow u \quad \text { and } \quad f_{i}\left(t_{k}, y_{t_{k}}\right) \rightarrow 0, \quad \text { as } \quad k \rightarrow \infty .
$$

For convenience, we prove it here.

Case 1. Assume that $y_{i}(t)$ is eventually monotone. In this case, $\lim _{t \rightarrow \infty} y_{i}(t)=u$ and, for $T$ large, either $\dot{y}_{i}(t) \leq 0$ for $t \geq T$ or $\dot{y}_{i}(t) \geq 0$ for $t \geq T$. Assume e.g. that $\dot{y}_{i}(t) \leq 0$ for all $t>T$ large (the situation $\dot{y}_{i}(t) \geq 0$ is analogous). Then $\dot{y}(t)=f_{i}\left(t, y_{t}\right) \leq 0$ for $t \geq T$ large, hence

$$
\limsup _{t \rightarrow \infty} f_{i}\left(t, y_{t}\right):=c \leq 0 .
$$

If $c<0$, then there is $t_{0}>0$ such that $f_{i}\left(t, y_{t}\right)<c / 2$ for $t \geq t_{0}$, implying that

$$
y_{i}(t)=y_{i}\left(t_{0}\right)+\int_{t_{0}}^{t} f_{i}\left(s, y_{s}\right) d s \leq y_{i}\left(t_{0}\right)+\frac{c}{2}\left(t-t_{0}\right),
$$

then $y_{i}(t) \rightarrow-\infty$ as $t \rightarrow \infty$, which is not possible because of (2.8). Thus $c=0$, which proves (2.9).

Case 2. Assume that $y_{i}(t)$ is not eventually monotone. In this case there is a sequence $\left(t_{k}\right)_{k \in \mathbb{N}}$ such that $t_{k} \nearrow \infty, \dot{y}_{i}\left(t_{k}\right)=0$ and $y_{i}\left(t_{k}\right) \rightarrow u$, as $k \rightarrow \infty$. Then $f_{i}\left(t_{k}, y_{t_{k}}\right)=0$ for all $k \in \mathbb{N}$, and (2.9) holds.

For the sake of contradiction, assume that $u>0$.

Fix $\varepsilon>0$ and let $T=T(\varepsilon)>0$ be such that $|y(t)|<u_{\epsilon}:=u+\varepsilon$, for $t \geq T$, and

$$
\int_{-\infty}^{-T} d \eta_{i j}^{(p)}(s)<\frac{\varepsilon}{\left\|y_{0}\right\|_{\infty}}, \quad i, j \in\{1, \ldots, n\}, p \in\{1, \ldots, P\} .
$$

Since $t-\tau_{i j}^{(p)}(t) \rightarrow \infty$ as $t \rightarrow \infty$ and $y_{i}\left(t_{k}\right) \rightarrow u>0$, then there is $k_{0} \in \mathbb{N}$ such that, for $k \geq k_{0}, t_{k}-\tau_{i j}^{(p)}\left(t_{k}\right)>T, t_{k}>2 T$, and $y_{i}\left(t_{k}\right)>0$. Hence, from the hypotheses and (2.8) we 
conclude that, for $k>k_{0}$,

$$
\begin{aligned}
& \bar{b}_{i}\left(y_{i}\left(t_{k}\right)+x_{i}^{*}\right)+h_{i}\left(t_{k}, y_{t_{k}}+x^{*}\right)= \\
& =\bar{b}_{i}\left(y_{i}\left(t_{k}\right)+x_{i}^{*}\right)-\bar{b}_{i}\left(x_{i}^{*}\right)+d_{i}^{-1} \sum_{j=1}^{n} \sum_{p=1}^{P}\left[\left(h_{i j}^{(p)}\left(d_{j} y_{j}\left(t_{k}-\tau_{i j}^{(p)}\left(t_{k}\right)\right)+d_{j} x_{j}^{*}\right)-h_{i j}^{(p)}\left(d_{j} x_{j}^{*}\right)\right)\right. \\
& \left.\quad+f_{i j}^{(p)}\left(\int_{-\infty}^{0} g_{i j}^{(p)}\left(d_{j} y_{j}\left(t_{k}+s\right)+d_{j} x_{j}^{*}\right) d \eta_{i j}^{(p)}(s)\right)-f_{i j}^{(p)}\left(g_{i j}^{(p)}\left(d_{j} x_{j}^{*}\right)\right)\right] \\
& \geq \beta_{i} y_{i}\left(t_{k}\right)-d_{i}^{-1} \sum_{j=1}^{n} \sum_{p=1}^{P}\left(\gamma_{i j}^{(p)} d_{j}\left|y_{j}\left(t_{k}-\tau_{i j}^{(p)}\left(t_{k}\right)\right)\right|+\mu_{i j}^{(p)} \sigma_{i j}^{(p)} d_{j} \int_{-\infty}^{0}\left|y_{j}\left(t_{k}+s\right)\right| d \eta_{i j}^{(p)}(s)\right) \\
& \geq \beta_{i} y_{i}\left(t_{k}\right)-d_{i}^{-1} \sum_{j=1}^{n} \sum_{p=1}^{P}\left[\gamma_{i j}^{(p)} d_{j} u_{\varepsilon}+\mu_{i j}^{(p)} \sigma_{i j}^{(p)} d_{j}\left(\int_{-\infty}^{-T}\left|y_{j}\left(t_{k}+s\right)\right| d \eta_{i j}^{(p)}(s)+\int_{-T}^{0}\left|y_{j}\left(t_{k}+s\right)\right| d \eta_{i j}^{(p)}(s)\right)\right] \\
& \geq \beta_{i} y_{i}\left(t_{k}\right)-d_{i}^{-1} \sum_{j=1}^{n} \sum_{p=1}^{P}\left[\gamma_{i j}^{(p)} d_{j} u_{\varepsilon}+\mu_{i j}^{(p)} \sigma_{i j}^{(p)} d_{j}\left(\varepsilon+u_{\varepsilon} \int_{-T}^{0} d \eta_{i j}^{(p)}(s)\right)\right] \\
& \geq \beta_{i} y_{i}\left(t_{k}\right)-d_{i}^{-1} \sum_{j=1}^{n} \sum_{p=1}^{P}\left(\gamma_{i j}^{(p)} d_{j} u_{\varepsilon}+\mu_{i j}^{(p)} \sigma_{i j}^{(p)} d_{j} u_{2 \varepsilon}\right) \geq \beta_{i} y_{i}\left(t_{k}\right)-d_{i}^{-1} \sum_{j=1}^{n} d_{j} l_{i j} u_{2 \varepsilon} .
\end{aligned}
$$

Letting $k \rightarrow \infty$ and $\varepsilon \rightarrow 0$, we get

$$
\liminf _{k \rightarrow \infty}\left[\bar{b}_{i}\left(y_{i}\left(t_{k}\right)+x_{i}^{*}\right)+h_{i}\left(t_{k}, y_{t_{k}}+x^{*}\right)\right] \geq\left(\beta_{i}-d_{i}^{-1} \sum_{j=1}^{n} l_{i j} d_{j}\right) u>0 .
$$

On the other hand, since $y_{i}(t)$ is bounded and $\bar{a}_{i}$ is positive and continuous, there is $K>0$ such that $\bar{a}_{i}\left(y_{i}(t)+x_{i}^{*}\right)>K$ for all $t \geq 0$. Together with the above inequality, this implies that $f_{i}\left(t_{k}, y_{t_{k}}\right) \nrightarrow 0$ as $k \rightarrow \infty$, which is a contradiction. Thus $u=0$, hence $v=0$ as well, and this ends the proof of the theorem.

Example 2.1. Consider the Cohen-Grossberg neural network model with infinite discrete time-varying delays (see [12])

$$
\dot{x}_{i}(t)=-a_{i}\left(x_{i}(t)\right)\left[b_{i}\left(x_{i}(t)\right)-\sum_{j=1}^{n} c_{i j} g_{j}\left(x_{j}(t)\right)-\sum_{j=1}^{n} d_{i j} f_{j}\left(x_{j}\left(t-\tau_{i j}(t)\right)\right)\right], i=1, \ldots, n
$$

where $c_{i j}, d_{i j} \in \mathbb{R}$ and $a_{i}: \mathbb{R} \rightarrow(0, \infty), b_{i}: \mathbb{R} \rightarrow \mathbb{R}$ and $\tau_{i j}:[0, \infty) \rightarrow[0, \infty)$ are continuous functions, $g_{i}, f_{i}: \mathbb{R} \rightarrow \mathbb{R}$ are Lipschitz functions, and $t-\tau_{i j}(t) \rightarrow \infty$ as $t \rightarrow \infty$, for $i, j=1, \ldots, n$. Note that system (2.10) includes known models of cellular neural networks as particular cases.

System (2.10) has the form (2.1) if $P=2, h_{i j}^{(1)}(u)=-c_{i j} g_{j}(u), h_{i j}^{(2)}(u)=-d_{i j} f_{j}(u)$, $f_{i j}^{(1)}(u)=f_{i j}^{(2)}(u)=0, \tau_{i j}^{(1)}(t)=0$ and $\tau_{i j}^{(2)}(t)=\tau_{i j}(t), u \in \mathbb{R}, t \geq 0, i, j=1, \ldots, n$. If 
$g_{i}, f_{i}: \mathbb{R} \rightarrow \mathbb{R}$ have Lipschitz constants $G_{i}, F_{i}$ respectively, then $h_{i j}^{(1)}, h_{i j}^{(2)}$ are also Lipschitz functions, with Lipschitz constants $l_{i j}^{(1)}=\left|c_{i j}\right| G_{j}, l_{i j}^{(2)}=\left|d_{i j}\right| F_{j}$ respectively, for all $i, j \in$ $\{1, \ldots, n\}$. Theorem 2.1 applied to system (2.10) gives the following result:

Corollary 2.2. Assume that (A1) holds, $\tau_{i j}:[0, \infty) \rightarrow[0, \infty)$ satisfy $t-\tau_{i j}(t) \rightarrow \infty$ as $t \rightarrow \infty$, and that $g_{i}, f_{i}: \mathbb{R} \rightarrow \mathbb{R}$ are Lipschitz functions with Lipschitz constants $G_{i}, F_{i}$, for all $i, j=1, \ldots, n$. If

$$
N:=B-L, \quad \text { where } \quad B=\operatorname{diag}\left(\beta_{1}, \ldots, \beta_{n}\right), L=\left[l_{i j}^{(1)}+l_{i j}^{(2)}\right],
$$

with $\beta_{i}$ as in (A1) and $l_{i j}^{(1)}=\left|c_{i j}\right| G_{j}, l_{i j}^{(2)}=\left|d_{i j}\right| F_{j}$, is a non-singular M-matrix, then there is a unique equilibrium point of (2.10), which is globally attractive.

Remark 2.1. For system (2.10), the global attractivity of an equilibrium point was already obtained by T. Huang et al. [12], provided that: the functions $f_{i}, g_{i}$ are Lipschitzian, the matrix $N$ as above is a non-singular M-matrix, $b_{i}(u)$ are differentiable functions such that $b_{i}^{\prime}(u) \geq \beta_{i}>0$ (clearly a stronger hypothesis than (A1)) and the following additional hypothesis is satisfied:

for each $i \in\{1, \ldots, n\}$, there exist $\underline{a}_{i}, \bar{a}_{i}>0$ such that $0<\underline{a}_{i} \leq a_{i}(u) \leq \bar{a}_{i}, u \in \mathbb{R}$.

Thus, the above Corollary 2.2 significantly improves the criterion in [12]. We also remark that the global exponential stability of the equilibrium point of (2.10), with bounded delays $\tau_{i j}$, was studied in $[6]$.

In the following example, a bidirectional associative memory (BAM) neural network model is treated as a particular case of the generalized Cohen-Grossberg model (2.1).

Example 2.2. Consider the BAM neural networks model presented in [15] (see also [1])

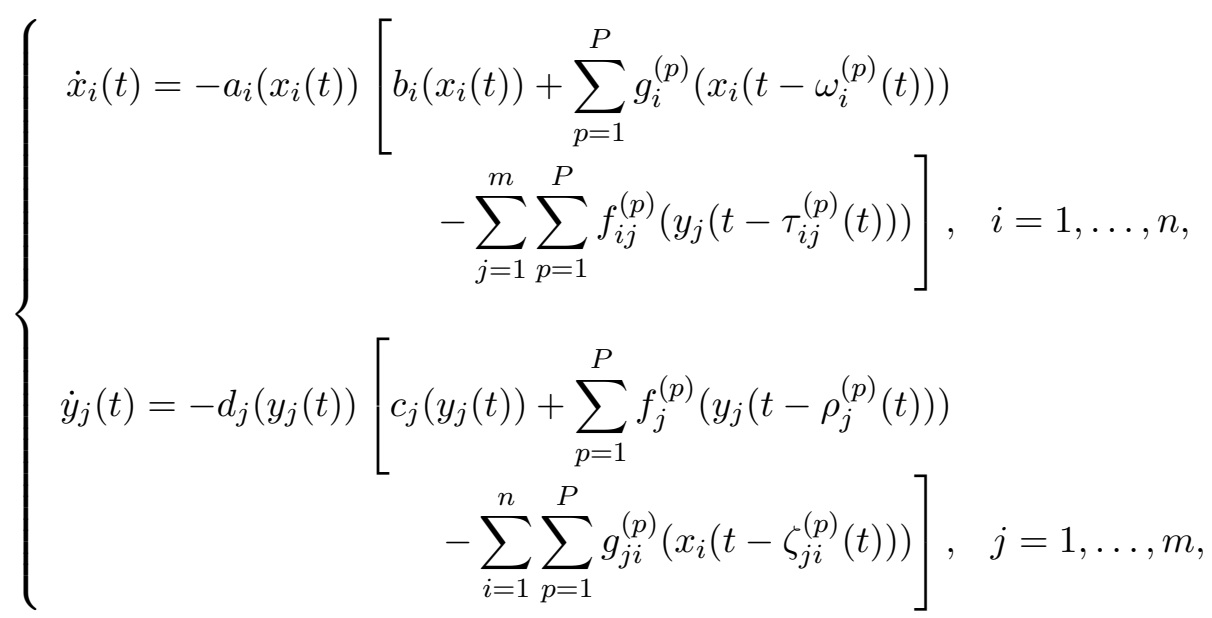

for $t \geq 0$ and $n, m, P \in \mathbb{N}$, where $a_{i}, d_{j}: \mathbb{R} \rightarrow(0, \infty), b_{i}, c_{j}, g_{i}^{(p)}, f_{j}^{(p)}, g_{j i}^{(p)}, f_{i j}^{(p)}: \mathbb{R} \rightarrow \mathbb{R}$ are continuous functions and $\omega_{i}^{(p)}, \rho_{j}^{(p)}, \tau_{i j}^{(p)}, \zeta_{j i}^{(p)}:[0, \infty) \rightarrow[0, \infty)$ are continuous functions such that $t-\omega_{i}^{(p)}(t) \rightarrow \infty, t-\rho_{j}^{(p)}(t) \rightarrow \infty, t-\tau_{i j}^{(p)}(t) \rightarrow \infty$, and $t-\zeta_{j}^{(p)}(t) \rightarrow \infty$ as $t \rightarrow \infty$, $i=1, \ldots, n, j=1, \ldots, m$ and $p=1, \ldots, P$. Contrarily to what happens in [15], we do not assume that the time-dependent delays $\omega_{i}^{(p)}(t), \rho_{j}^{(p)}(t), \tau_{i j}^{(p)}(t), \zeta_{j i}^{(p)}(t)$ are bounded.

Clearly, (2.12) is a particular case of (2.1). Next result is a direct consequence of Theorem 2.1 , and extends the stability criterion presented in [15] to the situation with unbounded discrete time-varying delays. 
Corollary 2.3. Suppose that $b_{i}$ and $c_{j}$ satisfy (A1) with constants $\beta_{i}$ and $\gamma_{j}$ respectively, $a_{i}(u)>0$ and $d_{j}(u)>0$ for all $u \in \mathbb{R}, f_{j}^{(p)}, g_{i}^{(p)}, f_{i j}^{(p)}, g_{j i}^{(p)}$ are Lipschitz functions with Lipschitz constants $\theta_{j}^{(p)}, \xi_{i}^{(p)}, \theta_{i j}^{(p)}, \xi_{j i}^{(p)}$ respectively, and $\omega_{i}^{(p)}, \rho_{j}^{(p)}, \tau_{i j}^{(p)}, \zeta_{j i}^{(p)}$ are continuous functions such that

$$
t-\min _{i, j, p}\left\{\omega_{i}^{(p)}(t), \rho_{j}^{(p)}(t), \tau_{i j}^{(p)}(t), \zeta_{j i}^{(p)}(t)\right\} \rightarrow \infty \quad \text { as } \quad t \rightarrow \infty .
$$

Define

$$
N:=\left[\begin{array}{cc}
B-G_{d} & -F \\
-G & C-F_{d}
\end{array}\right]_{(n+m) \times(n+m)}
$$

where

$$
\begin{gathered}
B=\operatorname{diag}\left(\beta_{1}, \ldots, \beta_{n}\right), \quad C=\operatorname{diag}\left(\gamma_{1}, \ldots, \gamma_{m}\right) \\
G_{d}=\operatorname{diag}\left(\sum_{p=1}^{P} \xi_{1}^{(p)}, \ldots, \sum_{p=1}^{P} \xi_{n}^{(p)}\right), \quad F_{d}=\operatorname{diag}\left(\sum_{p=1}^{P} \theta_{1}^{(p)}, \ldots, \sum_{p=1}^{P} \theta_{m}^{(p)}\right), \\
G=\left[\sum_{p=1}^{P} \xi_{j i}^{(p)}\right]_{m \times n}, \quad F=\left[\sum_{p=1}^{P} \theta_{i j}^{(p)}\right]_{n \times m} .
\end{gathered}
$$

If $N$ is a non-singular M-matrix, then there is a unique equilibrium point of (2.12), which is globally attractive.

\section{Exponential stability}

In this section, we address the global exponential stability of the equilibrium point of (2.1). Here system (2.1) is considered in the phase space $U C_{g}$, with $g(s)=e^{-\alpha s}, s \in(-\infty, 0]$, for a convenient $\alpha>0$ defined below, equipped with the norm $\|\phi\|_{g, c}=\sup _{s \leq 0} \frac{|\phi(s)|_{\infty, c}}{g(s)}$, where $|\cdot|_{\infty, c}$ is the norm in $\mathbb{R}^{n}$ defined by $|y|_{\infty, c}=\left|\left(y_{1}, \ldots, y_{n}\right)\right|_{\infty, c}=\max _{i}\left\{c_{i}\left|y_{i}\right|\right\}$ with $c=\left(c_{1}, \ldots, c_{n}\right)>0$.

The general family of functional differential systems in the phase space $U C_{g}$,

$$
\dot{x}_{i}(t)=-\rho_{i}\left(t, x_{t}\right)\left[b_{i}\left(x_{i}(t)\right)+f_{i}\left(t, x_{t}\right)\right], \quad i=1, \ldots, n, t \geq 0,
$$

where $\rho_{i}:[0,+\infty) \times U C_{g} \rightarrow(0,+\infty), b_{i}: \mathbb{R} \rightarrow \mathbb{R}$, and $f_{i}:[0,+\infty) \times U C_{g} \rightarrow \mathbb{R}$ are continuous functions, was considered in [7]. For system (3.1), the following set of hypotheses was imposed:

(E1) for each $i \in\{1, \ldots, n\}$, there is $\beta_{i}>0$ such that

$$
\left(b_{i}(u)-b_{i}(v)\right) /(u-v) \geq \beta_{i}, \quad \forall u, v \in \mathbb{R}, u \neq v ;
$$

(E2) for each $i \in\{1, \ldots, n\}$, there is $l_{i}>0$ such that

$$
\left|f_{i}(t, \varphi)-f_{i}(t, \psi)\right| \leq l_{i}|| \varphi-\psi \|_{g, c} \quad \forall \varphi, \psi \in U C_{g} ;
$$


(E3)

$\underline{\rho}:=\inf \left\{\rho_{i}(t, \varphi): t \geq 0, \varphi \in B C_{g}, i=1, \ldots, n\right\}>0$

(E4) for each $i \in\{1, \ldots, n\}, \beta_{i}>c_{i} l_{i}$.

In [7], the following result was established.

Theorem 3.1. [7] Assume (E1)-(E4).

If $x^{*}=\left(x_{1}^{*}, \ldots, x_{n}^{*}\right) \in \mathbb{R}^{n}$ is an equilibrium point of (3.1), then there are positive constants $\varepsilon, M$ such that

$$
\left|x(t, 0, \varphi)-x^{*}\right|_{\infty, c} \leq M e^{-\varepsilon t}|| \varphi-x^{*} \|_{\infty, c}, \quad \forall t \geq 0, \varphi \in B C_{g} .
$$

In order to apply the exponential stability criterion presented in the previous theorem to model (2.1), we now assume that the delayed functions $\tau_{i j}^{(p)}(s)$ are bounded, i.e., $0 \leq \tau_{i j}^{(p)}(t) \leq$ $\tau$ for some $\tau>0$, and there exists $\xi>0$ such that all the normalized non-decreasing and bounded functions $\eta_{i j}^{(p)}$ satisfy

$$
\int_{-\infty}^{0} e^{-\xi s} d \eta_{i j}^{(p)}(s)<\infty
$$

Theorem 3.2. Consider (2.1), where $a_{i}: \mathbb{R} \rightarrow(0,+\infty), b_{i}: \mathbb{R} \rightarrow \mathbb{R}$, and $\tau_{i j}^{(p)}:[0,+\infty) \rightarrow$ $[0,+\infty)$ are continuous, $h_{i j}^{(p)}, f_{i j}^{(p)}$, and $g_{i j}^{(p)}$ are Lipschitz functions with Lipschiz constants $\gamma_{i j}^{(p)}, \mu_{i j}^{(p)}$, and $\sigma_{i j}^{(p)}$ respectively, and $\eta_{i j}^{(p)}$ are non-decreasing, bounded and normalized, i.e. $\eta_{i j}^{(p)}(0)-\eta_{i j}^{(p)}(-\infty)=1, i, j=1, \ldots, n, p=1, \ldots, P$.

Assume in addition that:

(i) (E1) is satisfied;

(ii) $\underline{a_{i}}:=\inf \left\{a_{i}(x): x \in \mathbb{R}\right\}>0$ for $i=1, \ldots, n$;

(iii) there exists a constant $\xi>0$ such that $\eta_{i j}^{(p)}$ satisfy (3.2) for all $i, j=1, \ldots, n$, $p=1, \ldots, P$;

(iv) there exists $\tau>0$ such that $0 \leq \tau_{i j}^{(p)}(t) \leq \tau$ for $t \geq 0, i, j=1, \ldots, n, p=1, \ldots, P$. If the matrix $N$ defined in (2.3) is a non-singular M-matrix, then there is a unique equilibrium point of (2.1), which is globally exponentially stable.

Proof. Since $N$ is a non-singular M-matrix, there is $d=\left(d_{1}, \ldots, d_{n}\right)>0$ such that $(2.4)$ holds (see [8]), hence there is $\delta>0$ such that

$$
\beta_{i}>d_{i}^{-1}\left(\sum_{j=1}^{n} l_{i j}(1+\delta) d_{j}\right), \quad i=1, \ldots, n .
$$

As in the proof of Theorem 4.3 in [7], from (3.2) we can conclude that there is $\varsigma \in(0, \xi)$ such that

$$
\int_{-\infty}^{0} e^{-\varsigma s} d \eta_{i j}^{(p)}(s)<1+\delta, \quad i, j=1, \ldots, n, p=1, \ldots, P
$$

Let $\alpha:=\min \left\{\varsigma, \frac{\log (1+\delta)}{\tau}\right\}$ and consider system (2.1) in the phase space $U C_{g}$, where $g(s)=e^{-\alpha s}, s \leq 0$, equipped with the norm $\|\phi\|_{g, c}$ with $c=\left(d_{1}^{-1}, \ldots, d_{n}^{-1}\right)$. From Theorem 
2.1 , we know that there exists a unique equilibrium point, $x^{*}=\left(x_{1}^{*}, \ldots, x_{n}^{*}\right) \in \mathbb{R}^{n}$. System (2.1) has the form (3.1) with

$$
f_{i}(t, \phi)=\sum_{j=1}^{n} \sum_{p=1}^{P}\left(h_{i j}^{(p)}\left(\phi_{j}\left(-\tau_{i j}^{(p)}(t)\right)\right)+f_{i j}^{(p)}\left(\int_{-\infty}^{0} g_{i j}^{(p)}\left(\phi_{j}(s)\right) d \eta_{i j}^{(p)}(s)\right)\right) .
$$

For $\varphi, \phi \in U C_{g}$ and $t \geq 0$, since $h_{i j}^{(p)}, f_{i j}^{(p)}, g_{i j}^{(p)}$ are Lipschitz functions and $\eta_{i j}^{(p)}$ are non-decreasing, we have

$$
\begin{aligned}
\mid & f_{i}(t, \phi)-f_{i}(t, \varphi) \mid= \\
= & \mid \sum_{j=1}^{n} \sum_{p=1}^{P}\left(h_{i j}^{(p)}\left(\phi_{j}\left(-\tau_{i j}^{(p)}(t)\right)\right)-h_{i j}^{(p)}\left(\varphi_{j}\left(-\tau_{i j}^{(p)}(t)\right)\right)\right. \\
& \left.+f_{i j}^{(p)}\left(\int_{-\infty}^{0} g_{i j}^{(p)}\left(\phi_{j}(s)\right) d \eta_{i j}^{(p)}(s)\right)-f_{i j}^{(p)}\left(\int_{-\infty}^{0} g_{i j}^{(p)}\left(\varphi_{j}(s)\right) d \eta_{i j}^{(p)}(s)\right)\right) \mid \\
\leq & \sum_{j=1}^{n} \sum_{p=1}^{P}\left(\gamma_{i j}^{(p)}\left|\phi_{j}\left(-\tau_{i j}^{(p)}(t)\right)-\varphi_{j}\left(-\tau_{i j}^{(p)}(t)\right)\right|+\mu_{i j}^{(p)}\left|\int_{-\infty}^{0}\left[g_{i j}^{(p)}\left(\phi_{j}(s)\right)-g_{i j}^{(p)}\left(\varphi_{j}(s)\right)\right] d \eta_{i j}^{(p)}(s)\right|\right) \\
\leq & \sum_{j=1}^{n} \sum_{p=1}^{P}\left(\gamma_{i j}^{(p)} d_{j} \frac{d_{j}^{-1}\left|\left(\phi_{j}-\varphi_{j}\right)\left(-\tau_{i j}^{(p)}(t)\right)\right|}{e^{\alpha \tau_{i j}^{(p)}(t)}} e^{\alpha \tau_{i j}^{(p)}(t)}+\mu_{i j}^{(p)} \sigma_{i j}^{(p)} \int_{-\infty}^{0} e^{-\alpha s} d_{j} \frac{d_{j}^{-1}\left|\left(\phi_{j}-\varphi_{j}\right)(s)\right|}{e^{-\alpha s}} d \eta_{i j}^{(p)}(s)\right) \\
\leq & \sum_{j=1}^{n} \sum_{p=1}^{P}\left(\gamma_{i j}^{(p)} e^{\alpha \tau_{i j}^{(p)}(t)} d_{j}+\mu_{i j}^{(p)} \sigma_{i j}^{(p)} d_{j} \int_{-\infty}^{0} e^{-\alpha s} d \eta_{i j}^{(p)}(s)\right)\|\phi-\varphi\|_{g, c} \\
\leq & \sum_{j=1}^{n} \sum_{p=1}^{P}\left(\gamma_{i j}^{(p)}(1+\delta) d_{j}+\mu_{i j}^{(p)} \sigma_{i j}^{(p)} d_{j}(1+\delta)\right)\|\phi-\varphi\|_{g, c} \leq\left(\sum_{j=1}^{n} l_{i j}(1+\delta) d_{j}\right)\|\phi-\varphi\|_{g, c} .
\end{aligned}
$$

This means that

$$
\left|f_{i}(t, \phi)-f_{i}(t, \varphi)\right| \leq l_{i}\|\phi-\varphi\|_{g, c}, \quad i=1, \ldots, n,
$$

with $l_{i}:=\sum_{j=1}^{n} l_{i j}(1+\delta) d_{j}$, and from (3.3) we have $\beta_{i}>c_{i} l_{i}, i \in\{1, \ldots, n\}$. Now, the conclusion follows from Theorem 3.1.

Remark 3.1 Since the non-autonomous terms $h_{i j}^{(p)}$ were not included in the family of neural network models studied in [7], the previous result improves Theorem 4.3 in [7].

Example 3.1. If we take $P=2, h_{i j}^{(1)}(u)=-c_{i j} g_{j}(u), f_{i j}^{(1)}(u)=0, \tau_{i j}^{(1)}(t)=0, \tau_{i j}^{(2)}(t)=$ $\tau_{i j}(t), h_{i j}^{(2)}(u)=-d_{i j} f_{j}(u), f_{i j}^{(2)}(u)=-q_{i j} u+I_{i}, g_{i j}^{(2)}(u)=v_{j}(u)$, with $c_{i j}, d_{i j}, q_{i j}, I_{i} \in \mathbb{R}$, and

$$
\eta_{i j}^{(2)}(s)=\int_{-\infty}^{s} k_{i j}(-v) d v, \quad s \in(-\infty, 0], \quad i, j=1, \ldots, n,
$$


where $k_{i j}:[0,+\infty) \rightarrow[0,+\infty)$ are continuous functions, the model (2.1) becomes the following Cohen-Grossberg neural networks model

$$
\begin{aligned}
\dot{x}_{i}(t)=-a_{i}\left(x_{i}(t)\right)\left[b_{i}\left(x_{i}(t)\right)-\sum_{j=1}^{n} c_{i j} g_{j}\left(x_{j}(t)\right)-\sum_{j=1}^{n} d_{i j} f_{j}\left(x_{j}\left(t-\tau_{i j}(t)\right)\right)\right. & \\
& \left.-\sum_{j=1}^{n} q_{i j} \int_{-\infty}^{0} k_{i j}(-s) v_{j}\left(x_{j}(t+s)\right) d s+I_{i}\right], \quad t \geq 0,
\end{aligned}
$$

for $i=1, \ldots, n$, with the connection matrices

$$
C=\left[c_{i j}\right]_{n \times n}, \quad D=\left[d_{i j}\right]_{n \times n}, \quad \text { and } \quad Q=\left[q_{i j}\right]_{n \times n} .
$$

Applying Theorem 3.2 to model (3.5), we have the following result.

Corollary 3.3. Consider (3.5), where $a_{i}: \mathbb{R} \rightarrow(0,+\infty), b_{i}: \mathbb{R} \rightarrow \mathbb{R}$, and $\tau_{i j}:[0,+\infty) \rightarrow$ $[0,+\infty)$ are continuous, $g_{j}, f_{j}$, and $v_{j}$ are Lipschitz functions with Lipschiz constants $G_{j}$, $F_{j}$, and $V_{j}$ respectively, and $k_{i j}$ are nonnegative continuous functions such that

$$
\int_{0}^{+\infty} k_{i j}(t) d t=1, \quad i, j=1, \ldots, n
$$

Assume in addition that:

(i) (E1) is satisfied;

(ii) $\underline{a_{i}}:=\inf \left\{a_{i}(x): x \in \mathbb{R}\right\}>0$ for $i=1, \ldots, n$;

(iii) there exists a constant $\xi>0$ such that

$$
\int_{0}^{+\infty} k_{i j}(t) e^{\xi t} d t<+\infty, \quad i, j=1, \ldots, n
$$

(iv) there exists $\tau>0$ such that $0 \leq \tau_{i j}^{(p)}(t) \leq \tau$ for $t \geq 0, i, j=1, \ldots, n, p=1, \ldots, P$. If the matrix

$$
N=B-|C| G-|D| F-|Q| V
$$

where $B=\operatorname{diag}\left(\beta_{1}, \ldots, \beta_{n}\right), G=\operatorname{diag}\left(G_{1}, \ldots, G_{n}\right), F=\operatorname{diag}\left(F_{1}, \ldots, F_{n}\right)$, and $V=$ $\operatorname{diag}\left(V_{1}, \ldots, V_{n}\right)$, is a non-singular $M$-matrix, then there is a unique equilibrium point of (3.5), which is globally exponentially stable.

Remark 3.2 Since model (3.5) is only a particular case of model (2.1), our Theorem 3.2 is more general than the main result in [16].

Example 3.2. Consider the following model:

$$
\left\{\begin{aligned}
\dot{x}(t)=- & (2+\cos x(t))\left[6 x(t)+g_{1}(x(t))+g_{1}(y(t))\right. \\
& \left.+g_{1}(x(t-\tau(t)))+\int_{-\infty}^{0} k(-s) y(t+s) d s\right] \\
\dot{y}(t)=-(2+\sin y(t))\left[4.5 y(t)+g_{2}(y(t))+g_{2}(x(t-\tau(t)))\right. & \\
& \left.+g_{2}(y(t-\tau(t)))+\int_{-\infty}^{0} k(-s) x(t+s) d s\right]
\end{aligned}\right.
$$


where $g_{1}(u)=\frac{1}{2}(|u+1|-|u-1|), g_{2}(u)=\frac{1}{2}(|u+1|+|u-1|), \tau(t)=3|\cos t|+1$, and $k(t)=e^{-t}$.

The model (3.6) satisfies all hypotheses of Theorem 3.2 with

$$
\underline{a_{1}}=\underline{a_{2}}=1, \quad \beta_{1}=6, \quad \beta_{2}=4.5, \quad \tau=4,
$$

and $g_{1}, g_{2}$ are Lipschitz functions with Lipschitz constant 1 . Thus,

$$
B=\left(\begin{array}{cc}
6 & 0 \\
0 & 4.5
\end{array}\right), \quad L=\left(\begin{array}{ll}
2 & 2 \\
2 & 2
\end{array}\right), \quad N=\left(\begin{array}{cc}
4 & -2 \\
-2 & 2.5
\end{array}\right)
$$

and it easy to see that $N$ is a non-singular M-matrix. From Theorem 3.2, we know that model (3.6) has a unique equilibrium point which is globally exponential stable.

Since there is not $a \in \mathbb{R}$ such that $g_{1}(u)=a g_{2}(u)$, for all $u \in \mathbb{R}$, the main result in [16] cannot be applied to prove the stability of model (3.6).

We used the Mathematica software to plot a numerical simulation of the behavior of the solution $(x(t), y(t))$ of model (3.6) with initial condition $\varphi(s)=(3 \cos s, 2 \sin s), s \leq 0$, for $\tau(t) \equiv 4$ and $k(t) \equiv 0$. Note that, in this simple case, the matrix $N=\left(\begin{array}{cc}4 & -1 \\ -1 & 2.5\end{array}\right)$ is also a non-singular M-matrix. Once again, we cannot apply the result in [16].

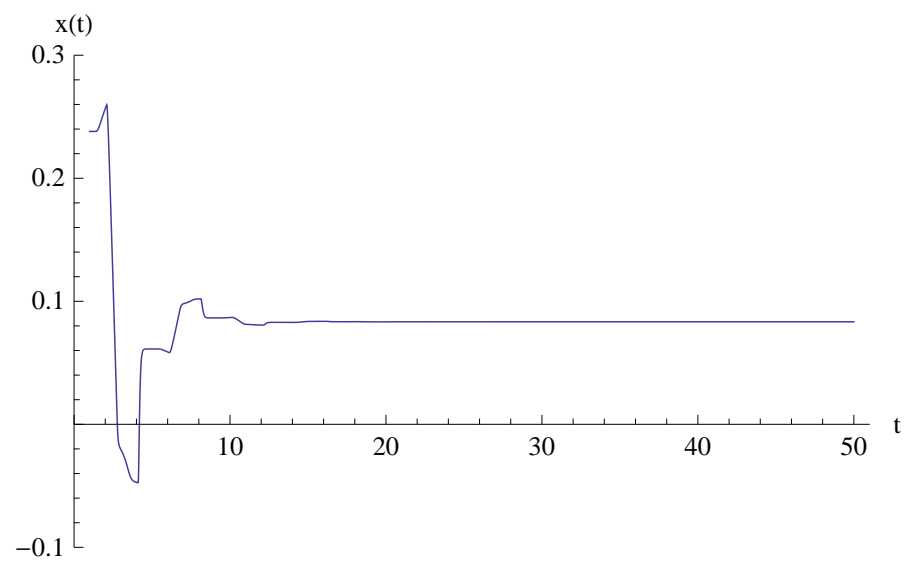

Figure 1: Behavior of the first neuron $x(t)$ in system (3.6) with $\tau(t) \equiv 4, k(t) \equiv 0$, and initial condition $\varphi(s)=(3 \cos s, 2 \sin s)$ 


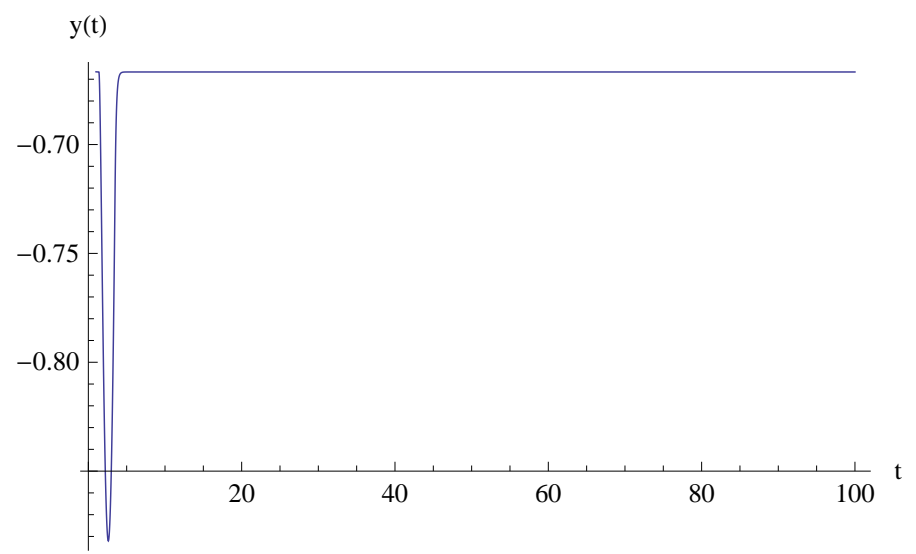

Figure 2: Behavior of the second neuron $y(t)$ in system (3.6) with $\tau(t) \equiv 4, k(t) \equiv 0$, and initial condition $\varphi(s)=(3 \cos s, 2 \sin s)$

Acknowledgments. This research was supported by FCT (Portugal), through the research center CMAT. The author thanks Professor Teresa Faria, for helpful discussions. The author also thanks the referee for valuable comments.

\section{References}

[1] J. Cao and Q. Song, Stability in Cohen-Grossberg-type bidirectional associative memory neural networks with time-varying delays, Nonlinearity 19 (2006) 1601-1617.

[2] Y. Chen, Global asymptotic stability of delayed Cohen-Grossberg neural networks, IEEE Trans. Circuits Syst. 53 (2006) 351-357.

[3] T. Chen and L. Rong, Robust global exponential stability of Cohen-Grossberg neural networks with time delays, IEEE Trans. Neural Netw. 15 (2004) 203-206.

[4] M. Cohen and S. Grossberg, Absolute stability of global pattern formation and parallel memory storage by competitive neural networks, IEEE Trans. Systems Man Cybernet. 13 (1983) 815-826.

[5] T. Faria and J.J. Oliveira, Local and global stability for Lotka-Volterra systems with distributed delays and instantaneous negative feedbacks. J. Differential Equations 244 (2008) 1049-1079.

[6] T. Faria and J.J. Oliveira, Boundedness and global exponential stability for delayed differential equations with applications, Appl. Math. Comput. 214 (2009) 487-496.

[7] T. Faria and J.J. Oliveira, General criteria for asymptotic and exponential stability of neural network models with unbounded delays, preprint

[8] M. Fiedler, Special Matriz and Their Applications in Numerical Mathematics, Martinus Nijhoff Publ. (Kluwer), Dordrecht, 1986.

[9] M. Forti and A. Tesi, New condition for global stability of neural networks with application to linear, quadratic programming problems, IEEE Trans. Circuits Syst. 42 (1995) 354-366. 
[10] J. Haddock and W. Hornor, Precompactness and convergence in norm of positive orbits in a certain fading memory space, Funkcial. Ekvac. 31 (1988) 349-361.

[11] J.K. Hale, J. Kato, Phase Space for Retarded Equations with Infinite Delay, Funkcialaj Ekvacioj, 21 (1978) 11-41.

[12] T. Huang, A. Chan, Y. Huang and J. Cao, Stability of Cohen-Grossberg neural networks with time-varying delays, Neural Networks, 20 (2007) 868-873.

[13] Y. Ji, X. Lou and B. Cui, Global output convergence of Cohen-Grossberg neural networks with both time-varying and distributed delays, Chaos Solitons \& Fractals, 40 (2009) 344-354.

[14] C.M. Marcus and R.M. Westervelt, Stability of analogy neural netwoeks with delay, Physics Reviews A 39 (1989) 347-359.

[15] J.J. Oliveira, Global asymptotic stability for neural network models with distributed delays, Math. Comput. Modelling 50 (2009) 81-91.

[16] Q. Song and J. Cao, Stability analysis of Cohen-Grossberg neural network with both time-varying and continuously distributed delays, J. Comput. Appl. Math. 197 (2006) 188-203.

[17] Q.K. Song and Z.J. Zhao, Global dissipativity of neural networks with both variable and unbounded delays, Chaos Solitons \& Fractals, 25 (2005) 393-401.

[18] L. Wang, Stability of Cohen-Grossberg neural networks with distributed delays, Appl. Math. Comput. 160 (2005) 93-110.

[19] L. Wang and X. Zou, Harmless delays in Cohen-Grossberg neural networks, Physica D 170 (2002) 162-173.

[20] L. Wu, B.T. Cui and X.Y. Lou, Global exponential stability of Cohen-Grossberg neural networks with distributed delays, Math. Comput. Modelling 47 (2008) 868-873.

[21] J.Y. Zhang, Y. Suda and T. Iwasa, Absolutely exponential stability of a class of neural networks with unbounded delay, Neural Networks 17(2004) 391-397. 